\title{
Analysis of Chaos in Current-Mode-Controlled DC Drive Systems
}

\author{
J. H. Chen, K. T. Chau, Member, IEEE, and C. C. Chan, Fellow, IEEE
}

\begin{abstract}
In this paper, chaotic behavior in current-mode-controlled dc drive systems has been analyzed. The key is to derive an iterative map that describes the nonlinear system dynamics. Analytical modeling of fundamental and subharmonic oscillations as well as their stability analysis are presented. The results show that the current-mode-controlled de drive systems generally exhibit chaotic behavior. To avoid the occurrence of chaos, the stable ranges of various system parameters are determined. Both computer simulation and experimental measurement are given to verify the theoretical analysis.
\end{abstract}

Index Terms-Bifurcation, chaos, current control, dc motor drives.

\section{INTRODUCTION}

D UE TO the switching operation of power electronic systems, the corresponding system dynamics are generally nonlinear [1]-[5]. Starting in the 1970's, many researchers have focused on using various small-signal approximation techniques to model these system dynamics. Although those small-signal models are useful to assess the local stability, they are ill suited to describe any nonlinear dynamics. Recently, the investigation into these nonlinear dynamics has received much interest, especially the chaotic analysis of switching dc-dc converters and their practical issues [3]-[17]. Focusing on the investigation of chaotic phenomena occurring in power electronic systems, the most attractive approach has been the iterative nonlinear mapping [7]. The one-dimensional mapping was successfully employed to derive the chaotic region of switching $\mathrm{dc}-\mathrm{dc}$ converters in which the load voltage was assumed as a constant-voltage sink [3]. However, this load voltage is usually nonconstant for practical switching dc-dc converters. In order to investigate the chaos of these practical converters, the corresponding two-dimensional mapping has recently been applied [10]-[13].

On the other hand, the investigation of chaos in industrial drive systems has been surprisingly rare [18]-[20]. Namely, the bifurcation and chaos in a pulsewidth modulation (PWM) inverter-fed induction drive system were investigated by using numerical simulation, whereas the chaos in a brushless de drive system was analyzed by approximately transforming it into a Lorenz system. Before 1997 [21], the analytical investigation into industrial drive systems was almost absent.

Manuscript received February 13, 1998; revised April 16, 1999. This work was supported in part by the Committee on Research and Conference Grants, University of Hong Kong, and the Hong Kong Research Grants Council Project HKU7128/99E.

The authors are with the Department of Electrical and Electronic Engineering, University of Hong Kong, Hong Kong (e-mail: ktchau @eee.hku.hk).

Publisher Item Identifier S 0278-0046(00)01327-7.
The current-mode control scheme offers more straightforward torque control than the voltage-mode control scheme, but is prone to chaos and instability [8], [9]. It is the purpose of this paper to investigate, both numerically and analytically, the chaotic behavior of current-mode-controlled dc drive systems. This analysis should not ignore the switching effect of power converters, nor accept the rough assumptions of constant-voltage sink and constant-current reference. Hence, the stable and chaotic ranges of system parameters can be obtained, so the occurrence of chaos can be avoided.

In Section II, background will be given to describe the fundamental concepts and key issues about chaos and chaotic systems. In Section III, a second-order current-mode-controlled dc drive system will be described and then modeled. The corresponding chaotic analysis will be presented in Section IV, which includes the formulation of system solution, the derivation of an iterative map, the analysis of periodic orbits, and the stability analysis. Based on the derived map, computer simulations will be performed by both numerical and analytical techniques in Section V. Finally, Section VI will be devoted to experimental studies.

\section{BACKGROUND}

Chaos can be defined as bounded steady-state behavior that is not an equilibrium point, not periodic, and not quasi-periodic [22]-[24]. It is characterized by a noise-like spectrum which has a continuous broad-band nature, and may have no discrete components. Another property of chaos is sensitive dependence on initial condition. As the initial state of a practical system can never be specified exactly, but only to within some tolerance, the long-term behavior of a chaotic system can never be predicted. Thus, a chaotic system is usually described as a deterministic system that exhibits random-like behavior. An in-depth tutorial on chaos for engineers can be found in [22].

In the past decades, chaos was misinterpreted in industrial practice: it was reckoned as a noise when the bounded range of chaotic behavior was narrow, whereas it was classified as an unstable phenomenon when the bounded range was wide. Starting in the late 1980's, chaos has been identified to be a real phenomenon in power electronics [3], [4]. Then, a number of investigations into chaotic behavior of power electronic systems, especially dc-dc converters, has been carried out [5]-[13]. Some recent investigations have also been extended to control and applications of chaos in dc-dc converters [14]-[17]. Moreover, some chaotic problems in industrial drive systems have recently been investigated [18]-[21].

What are the practical issues of chaos? As mentioned before, chaos used to be misinterpreted as noisy or unstable operation 
of power electronic systems, including dc-dc converters and dc drives, in industrial practice. For examples, the output voltage oscillation of $\mathrm{dc}-\mathrm{dc}$ converters and the torque fluctuation of $\mathrm{dc}$ drives may result from chaotic operation due to the change of system parameters with respect to temperatures. If these chaotic systems are well explained and even predicted, they can be engineered. Thus, engineers can design those systems with parameters avoiding any chaotic operation. On the other hand, by regarding chaos as alternative modes of operation, chaotic power electronic systems can be utilized for some engineering applications. For example, the feasibility of using chaos to depress the spectral peaks of the interference from a dc-dc converter has been demonstrated [16]; and the use of chaos to jump rapidly between two stabilized operating modes of a dc-dc converter has been presented [17].

The fundamental concept for analyzing chaotic systems is due to Poincaré. It replaces the solution of a continuous-time dynamic system with an iterative map, the so-called the Poincaré map. This map acts like a stroboscope, which produces a sequence of samples of the continuous-time solution. Differing from classical discretization, the sampling may not occur at a regular interval, but at a specified instant such as the maximum or minimum value of the continuous-time solution. Hence, the steady-state behavior of the Poincare map, the so-called orbit, corresponds to the steady-state waveform of the continuous-time system. Hence, the phase-plane orbit of the Poincaré map, the so-called Poincaré section, corresponds to the trajectory at the same phase plane of the continuous-time system. The graphical representation of this Poincare section can provide insight into the system behavior. Namely, a fixed point of the Poincaré map represents a periodic steady-state solution, usually termed a period-1 orbit, whereas a closed orbit with $p$ points, called a period- $p$ orbit, is a periodic subharmonic solution with a frequency at $1 / p$ of the excitation frequency. In general, the Poincaré section during chaos appears as an intricate, multilayered, peculiar structure. Moreover, the stability of a periodic solution (a fixed point of the Poincaré map) is determined by its characteristic multipliers, also called Floquet multipliers, which are the eigenvalues of the Jacobian matrix of the Poincaré map at the fixed point. If all its characteristic multipliers lie within the unit circle at the complex plane, the fixed point is stable.

For a chaotic system, it is essential to know the formation of chaos due to the variation in system parameters. As a parameter is varied, a bifurcation is an abrupt change in the steady-state behavior of the system. A plot of the steady-state orbit against a bifurcation parameter is termed a bifurcation diagram. Thus, the resulting bifurcation diagrams facilitate the appraisal of the steady-state system behavior at a glance.

\section{SySTEM MODELING}

In this paper, the most commonly used dc drive system, namely the dc buck-chopper-fed permanent-magnet dc motor drive, is adopted for study. The corresponding schematic and equivalent circuit is shown in Fig. 1.
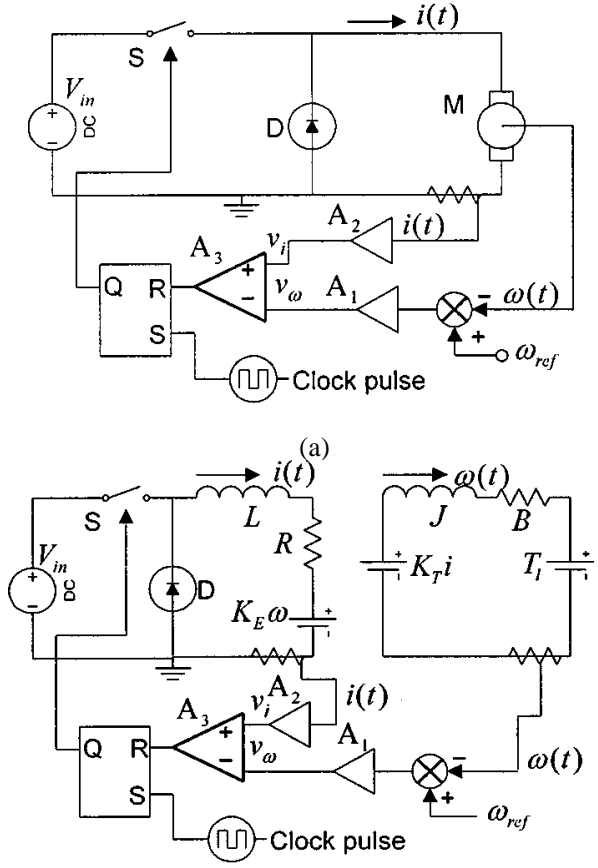

(b)

Fig. 1. Current-mode-controlled dc drive system. (a) Schematic. (b) Equivalent circuit.

Considering that the operational amplifier $A_{1}$ and $A_{2}$ have gains $g_{\omega}$ and $g_{i}$, the speed and current control signals $v_{\omega}$ and $v_{i}$ can be expressed as

$$
\begin{aligned}
& v_{\omega}(t)=g_{\omega}\left(\omega_{\mathrm{ref}}-\omega(t)\right) \\
& v_{i}(t)=g_{i} i(t)
\end{aligned}
$$

where

$i(t)$ armature current of the dc motor;

$\omega(t)$ rotor speed of the dc motor;

$\omega_{\text {ref }}$ reference speed of the dc motor.

Then, both $v_{\omega}$ and $v_{i}$ are fed into the comparator $A_{3}$ which outputs the pulse to the reset of an $R-S$ latch. The power switch $S$ is controlled by this $R-S$ latch which is set by clock pulses of period $T$. Once the latch is set by the clock pulse, $S$ is turned on and diode $D$ is off. Then, $S$ keeps closed until $v_{i}$ exceeds $v_{\omega}$ where the latch begins to reset. When the latch is reset, $S$ is turned off and $D$ is on. Then, $S$ remains open until the arrival of the next clock pulse where it closes again. If both set and reset signals occur simultaneously, the reset will dominate the set so that $S$ keeps open until the occurrence of another clock pulse. Therefore, the system equation can be divided into two stages as given by the following:

- stage 1 (setting the latch)

$$
\begin{aligned}
\frac{d}{d t} & \left(\begin{array}{c}
\omega(t) \\
i(t)
\end{array}\right) \\
& =\left(\begin{array}{cc}
-B / J & K_{T} / J \\
-K_{E} / L & -R / L
\end{array}\right)\left(\begin{array}{c}
\omega(t) \\
i(t)
\end{array}\right)+\left(\begin{array}{c}
-T_{l} / J \\
V_{\mathrm{in}} / L
\end{array}\right)
\end{aligned}
$$


- stage 2 (resetting the latch at $\left.v_{i}=v_{\omega}\right)$

$$
\begin{aligned}
& \frac{d}{d t}\left(\begin{array}{c}
\omega(t) \\
i(t)
\end{array}\right) \\
& \quad=\left(\begin{array}{cc}
-B / J & K_{T} / J \\
-K_{E} / L & -R / L
\end{array}\right)\left(\begin{array}{c}
\omega(t) \\
i(t)
\end{array}\right)+\left(\begin{array}{c}
-T_{l} / J \\
0
\end{array}\right)
\end{aligned}
$$

where

$R \quad$ armature resistance;

$L$ armature inductance;

$V_{\text {in }}$ dc supply voltage;

$K_{E}$ back-EMF constant;

$K_{T}$ torque constant;

$B$ viscous damping;

$J \quad$ load inertia;

$T_{l}$ load torque.

By defining the state vector $\boldsymbol{X}(t)$ and the matrices $\boldsymbol{A}, \boldsymbol{E}_{1}, \boldsymbol{E}_{2}, \boldsymbol{E}_{3}, \boldsymbol{E}_{4}$ as

$$
\begin{aligned}
X(t) & =\left(\begin{array}{c}
\omega(t) \\
i(t)
\end{array}\right) \\
A & =\left(\begin{array}{cc}
-B / J & K_{T} / J \\
-K_{E} / L & -R / L
\end{array}\right) \\
\boldsymbol{E}_{1} & =\left(\begin{array}{c}
-T_{l} / J \\
V_{\mathrm{in}} / L
\end{array}\right) \\
\boldsymbol{E}_{2} & =\left(\begin{array}{c}
-T_{l} / J \\
0
\end{array}\right) \\
\boldsymbol{E}_{3} & =\left(\begin{array}{c}
g_{\omega} \\
g_{i}
\end{array}\right) \\
\boldsymbol{E}_{4} & =\left(\begin{array}{c}
\omega_{\mathrm{ref}} \\
0
\end{array}\right)
\end{aligned}
$$

the system equation given by (3) and (4) can be rewritten as

$$
\dot{\boldsymbol{X}}(t)=\boldsymbol{A X}(t)+\boldsymbol{E}_{k}, \quad k=1,2 .
$$

By using (1) and (2), the switching condition $v_{i}(t)-v_{\omega}(t)=0$ can be expressed as

$$
E_{3}\left(\boldsymbol{X}(t)-E_{4}\right)=0 .
$$

It should be noted that (7) is a time-varying linear state equation switching between two stages. As its switching condition given by (8) depends on the external speed reference and the internal state vector, the whole system described by both (7) and (8) exhibits nonlinear dynamics.

\section{CHAOTIC ANALYSIS}

The analysis of system chaotic behavior begins with the solution of (7) in continuous-time domain, namely, $\boldsymbol{X}(t)$. Then, the system solution is described by a sequence of samples $\left\{\boldsymbol{X}_{n}\right\}$ $n=0,1,2, \cdots$. Differing from conventional discretization of a continuous-time state equation, the successive sample $\boldsymbol{X}_{n+1}$ may not be taken at $(n+1) T$. Sample $X_{n+1}$ occurs at $(n+m) T$ when there is a change of switching state after $m(m \geq 1)$ clock pulses, because there is no intersection between $v_{\omega}$ and $v_{i}$ within $(m-1)$ clock pulses [7]. The corresponding mapping from $\boldsymbol{X}_{n}$ to its successive sample $\boldsymbol{X}_{n+1}$ is termed the Poincaré map. Hence, the Poincaré map that maps a sample $\boldsymbol{X}_{n}$ of $\boldsymbol{X}(t)$ at $t=n T$ to its successive one $X_{n+1}$ at $t=(n+m) T$ is defined as $P: \Re^{2} \rightarrow \Re^{2}$

$$
\boldsymbol{X}_{n+1}=P\left(\boldsymbol{X}_{n}\right) .
$$

It should be noted that the Poincaré map given by (9) is a generalized case with $m \geq 1$, which can fully describe the system behavior. When $m>1$, the solution of (9) can only be solved by using numerical simulation. On the other hand, focusing on a specific case with $m=1$, the solution can be analytically solved so that the system behavior can be described in terms of periodic orbits and stability.

\section{A. Solution of System Equation}

Given an initial value $\boldsymbol{X}\left(t_{0}\right)$, the continuous-time solution of the system equation given by (7) can be expressed as

$$
\begin{gathered}
\boldsymbol{X}(t)=\Phi\left(t-t_{0}\right) \boldsymbol{X}\left(t_{0}\right)+\int_{t_{0}}^{t} \Phi(t-\tau) \boldsymbol{E}_{k} d \tau \\
k=1,2 .
\end{gathered}
$$

For a practical dc drive system, $\boldsymbol{A}$ given by (5) always yields a positive $\operatorname{det} A=\left(B R+K_{E} K_{T}\right) /(L J)$ and, hence, is always invertible. Moreover, even ignoring the positive $B R$ term, $\left(K_{E} K_{T}\right) /(L J)$ can be written as $1 /\left(\tau_{m} \tau_{a}\right)$ where $\tau_{m}=(J R) /\left(K_{E} K_{T}\right)$ is the mechanical time constant and $\tau_{a}=L / R$ is the electrical time constant [25]. For practical dc drive systems, as $\tau_{m}$ is from tens of milliseconds to several seconds and $\tau_{a}$ is from tens of microseconds to tens of milliseconds, $1 /\left(\tau_{m} \tau_{a}\right)$ and, hence, det $\boldsymbol{A}$ are seldom close to zero. Thus, the continuous-time solution given by (10) can be rewritten as

$$
\begin{gathered}
\boldsymbol{X}(t)=-\boldsymbol{A}^{-1} \boldsymbol{E}_{k}+\Phi\left(t-t_{0}\right)\left(\boldsymbol{X}\left(t_{0}\right)+\boldsymbol{A}^{-1} \boldsymbol{E}_{k}\right), \\
k=1,2
\end{gathered}
$$

where $\Phi(t)=e^{A t}$ is the state transition matrix. By defining the parameters $\alpha$ and $\Delta$ as

$$
\begin{aligned}
\alpha & =\frac{1}{2}\left(\frac{R}{L}+\frac{B}{J}\right) \\
\Delta & =\frac{1}{4}\left(\frac{R}{L}-\frac{B}{J}\right)^{2}-\frac{K_{E} K_{T}}{L J}
\end{aligned}
$$

the eigenvalues $\lambda_{1}, \lambda_{2}$ of $\boldsymbol{A}$ can be expressed as

$$
\begin{array}{ll}
\Delta=0, & \lambda_{1}=\lambda_{2}=\lambda=-\alpha \\
\Delta>0, & \lambda_{1}=-\alpha+\sqrt{\Delta}, \quad \lambda_{2}=-\alpha-\sqrt{\Delta} \\
\Delta<0, & \lambda_{1}=-\alpha+j \sqrt{-\Delta}, \quad \lambda_{2}=-\alpha-j \sqrt{-\Delta} .
\end{array}
$$

Hence, the corresponding $\Phi(t)$ can be obtained as

$$
\begin{aligned}
\Delta & =0, \quad \Phi(t)=\exp (-\alpha t)[1-t(2 \alpha 1-\boldsymbol{A})] \\
\Delta & >0, \\
\Phi(t) & =\frac{1}{\lambda_{2}-\lambda_{1}}\left[\exp \left(\lambda_{1} t\right)\left(\lambda_{2} 1-\boldsymbol{A}\right)-\exp \left(\lambda_{2} t\right)\left(\lambda_{1} 1-\boldsymbol{A}\right)\right]
\end{aligned}
$$

$\Delta<0$,

$$
\Phi(t)=\exp (-\alpha t)\left[1 \cos (\beta t)+\frac{1}{\beta}(\alpha 1+\boldsymbol{A}) \sin (\beta t)\right]
$$

where 1 is the identity matrix and $\beta=\sqrt{-\Delta}$. 


\section{B. Derivation of Generalized Poincaré Map}

Since $\boldsymbol{X}_{n}$ is always sampled at the beginning of the clock pulse that makes $S$ from off to on, hence, the drive system always operates in Stage 1 first and then in Stage 2 for each sampling interval. By defining the intervals of Stage 1 and Stage 2 as $\delta T$ and $\delta^{\prime} T$, respectively, the interval of Poincaré map becomes $m T=\left(\delta+\delta^{\prime}\right) T$. Thus, $\boldsymbol{X}(\delta T)$ and $\boldsymbol{X}_{n+1}$ can be directly deduced from (11) as given by

$$
\begin{aligned}
& \boldsymbol{X}(\delta T)=-\boldsymbol{A}^{-1} \boldsymbol{E}_{1}+\Phi(\delta T)\left(\boldsymbol{X}_{n}+\boldsymbol{A}^{-1} \boldsymbol{E}_{1}\right) \\
& \boldsymbol{X}_{n+1}=-\boldsymbol{A}^{-1} \boldsymbol{E}_{2}+\Phi(m T-\delta T)\left(\boldsymbol{X}(\delta T)+\boldsymbol{A}^{-1} \boldsymbol{E}_{2}\right) .
\end{aligned}
$$

By substituting (19) into (8), $\delta$ can be determined based on the solution of the following transcendental equation:

$$
h(\delta)=\boldsymbol{E}_{3}\left(-\boldsymbol{A}^{-1} \boldsymbol{E}_{1}+\Phi(\delta T)\left(\boldsymbol{X}_{n}+\boldsymbol{A}^{-1} \boldsymbol{E}_{1}\right)-\boldsymbol{E}_{4}\right)=0 .
$$

On the other hand, $m$ can be deduced as the minimum integer that is larger than $\delta$ while fulfilling $h(\delta)<0$. Hence, the Poincaré map can written as

$$
\begin{aligned}
\boldsymbol{X}_{n+1}=-\boldsymbol{A}^{-1} \boldsymbol{E}_{1}+ & \Phi(m T)\left(\boldsymbol{X}_{n}+\boldsymbol{A}^{-1} \boldsymbol{E}_{1}\right) \\
& +(1-\Phi(m T-\delta T)) \boldsymbol{A}^{-1}\left(\boldsymbol{E}_{1}-\boldsymbol{E}_{2}\right) .
\end{aligned}
$$

It should be noted that the derived Poincaré map is a generalized mapping which covers all possible solutions such as real and complex roots due to different system parameters and operating conditions. Thus, this generalized Poincaré map can be considered as the mapping for any second-order dynamical systems using similar mathematical models, such as other currentmode-controlled switching power converters and dc drive systems. Moreover, the derivation can readily be extended to those higher order dynamical systems involving power switches.

\section{Analysis of Periodic Orbits}

A natural numerical tool to obtain the steady-state solution of the generalized Poincaré map is the so-called brute-force method [22], [23] - repeating the iteration of the map until the transient has died out or the steady state has been reached. This method has the advantage of simplicity, but may suffer from tedious simulation due to long-lived transients. If $K$ iterations are spent for transient operation, $N$ points are needed to describe an orbit and $M$ steps are used to depict a bifurcation diagram, the total number of iterations for constructing a bifurcation diagram will be $(K+N) M$.

Based on the above generalized Poincaré map, dynamic bifurcation of the drive system can readily be investigated by employing the brute-force method. Thus, different bifurcation diagrams with respect to different system parameters can be obtained. However, as each iterative computation of the generalized Poincaré map needs to solve the transcendental equation, the corresponding numerical simulation is usually very tedious. In order to avoid the lengthy computation and to attain an insight into the periodic solution, the analysis can be focused on the case that the interval of mapping is the same as the clock cycle, mathematically $m=1$. The corresponding mapping is named as the specific Poincaré map.
The steady-state periodic solution of the drive system can be a period-1 orbit $\boldsymbol{X}^{*}$, or a period- $p$ orbit $\left\{X_{1}^{*}, \cdots, \boldsymbol{X}_{p}^{*}\right\}$ with $p>1$. The corresponding specific Poincaré maps are described as

$$
\begin{aligned}
\boldsymbol{X}^{*} & =P\left(\boldsymbol{X}^{*}\right) \\
\boldsymbol{X}_{k+1}^{*} & =P\left(\boldsymbol{X}_{k}^{*}\right), \quad k=1, \cdots, p-1 \\
\boldsymbol{X}_{1}^{*} & =P\left(\boldsymbol{X}_{p}^{*}\right) .
\end{aligned}
$$

First, the period- 1 orbit is analyzed. Since $m=1$, the corresponding Poincaré map can be obtained from (22) as given by

$$
\begin{aligned}
\boldsymbol{X}_{n+1}=-A^{-1} \boldsymbol{E}_{1} & +\Phi(T)\left(\boldsymbol{X}_{n}+\boldsymbol{A}^{-1} \boldsymbol{E}_{1}\right) \\
& +(1-\Phi(T-\delta T)) \boldsymbol{A}^{-1}\left(\boldsymbol{E}_{1}-\boldsymbol{E}_{2}\right)
\end{aligned}
$$

Substituting (25) into the mapping given by (23), the period-1 orbit can be deduced as

$$
\begin{array}{r}
\boldsymbol{X}^{*}=-\boldsymbol{A}^{-1} \boldsymbol{E}_{1}+(1-\Phi(T))^{-1}(1-\boldsymbol{\Phi}(T-\delta T)) \\
\cdot \boldsymbol{A}^{-1}\left(\boldsymbol{E}_{1}-\boldsymbol{E}_{2}\right)
\end{array}
$$

After substitution of (26) into (21), $\delta$ can be determined from the corresponding transcendental equation

$$
h(\delta)=\boldsymbol{E}_{3}\left[-A^{-1} \boldsymbol{E}_{1}+\Phi(\delta T)(1-\Phi(T))^{-1}(1-\Phi(T-\delta T))\right.
$$$$
\left.\cdot A^{-1}\left(\boldsymbol{E}_{1}-\boldsymbol{E}_{2}\right)-\boldsymbol{E}_{4}\right]=0 \text {. }
$$

Hence, provided that $\delta \in(0,1), \boldsymbol{X}^{*}$ can be obtained from (26).

Second, the period- $p$ orbit $\left\{X_{1}^{*}, \cdots, \boldsymbol{X}_{p}^{*}\right\}$ is analyzed. By defining $\delta_{1}, \cdots, \delta_{p}$ as the $p$ duty cycles within $p$ periods of clock pulses with $p>1$, the $p$-fold iterative mapping can be formulated from (25) as

$$
\begin{aligned}
\boldsymbol{X}_{n+p}= & P^{(p)}\left(\boldsymbol{X}_{n}\right) \\
= & -\boldsymbol{A}^{-1} \boldsymbol{E}_{1}+\Phi(p T)\left(\boldsymbol{X}_{n}+\boldsymbol{A}^{-1} \boldsymbol{E}_{1}\right) \\
& +\sum_{j=1}^{p}\left[\Phi((p-j) T)\left(1-\Phi\left(T-\delta_{j} T\right)\right)\right] \\
& \cdot \boldsymbol{A}^{-1}\left(\boldsymbol{E}_{1}-\boldsymbol{E}_{2}\right) .
\end{aligned}
$$

By using (24), the period- $p$ orbit can be obtained as

$$
\begin{aligned}
\boldsymbol{X}_{1}^{*}= & -A^{-1} \boldsymbol{E}_{1}+(1-\Phi(p T))^{-1} \sum_{j=1}^{p} \\
& \cdot\left[\Phi((p-j) T)\left(1-\Phi\left(T-\delta_{j} T\right)\right)\right] \boldsymbol{A}^{-1}\left(\boldsymbol{E}_{1}-\boldsymbol{E}_{2}\right)
\end{aligned}
$$

$$
\begin{aligned}
\boldsymbol{X}_{i}^{*}= & -\boldsymbol{A}^{-1} \boldsymbol{E}_{1}+\Phi((i-1) T)\left(\boldsymbol{X}_{1}^{*}+\boldsymbol{A}^{-1} \boldsymbol{E}_{1}\right) \\
& +\sum_{j=1}^{i-1}\left[\Phi((i-j-1) T)\left(1-\Phi\left(T-\delta_{j} T\right)\right)\right] A^{-1} \\
& \cdot\left(\boldsymbol{E}_{1}-\boldsymbol{E}_{2}\right), \quad i=2, \cdots, p \\
h_{i}(\boldsymbol{d})= & \boldsymbol{E}_{3}\left[-\boldsymbol{A}^{-1} \boldsymbol{E}_{1}+\Phi\left(\delta_{i} T\right)\left(\boldsymbol{X}_{i}^{*}+\boldsymbol{A}^{-1} \boldsymbol{E}_{1}\right)-\boldsymbol{E}_{4}\right]=0 \\
& \quad i=1, \cdots, p
\end{aligned}
$$

where $\Phi(0)=1$ and $\boldsymbol{d}=\left(\delta_{1}, \cdots, \delta_{p}\right)$. By substituting (29) and (30) into (31), $\boldsymbol{d}$ can be determined. Hence, provided that $\delta_{i} \in$ 


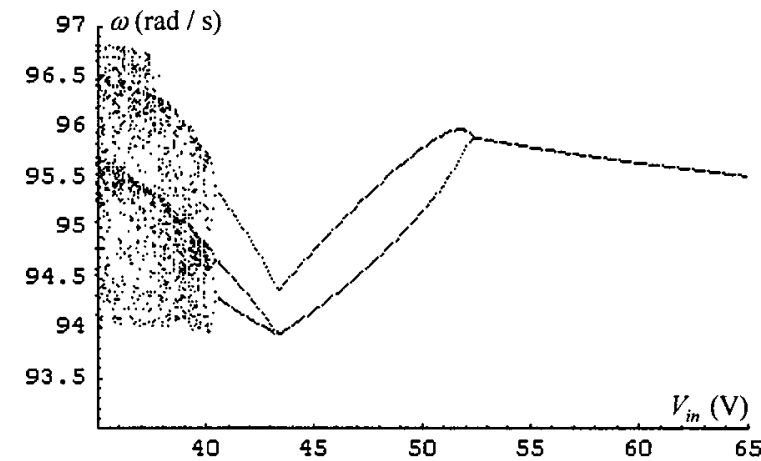

(a)

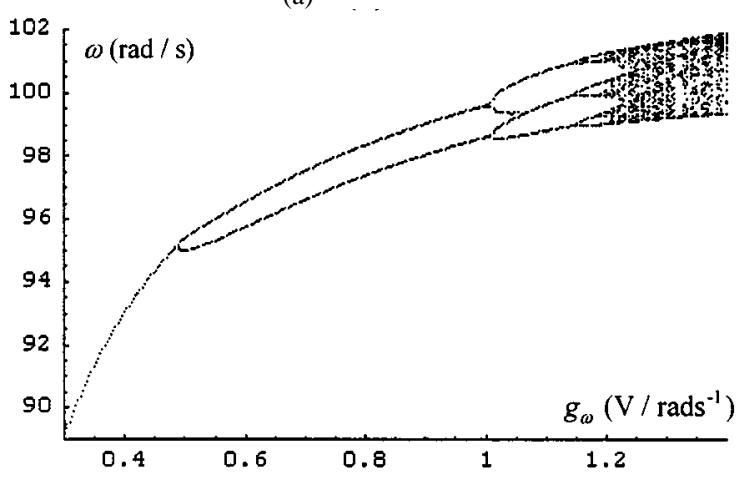

(c)

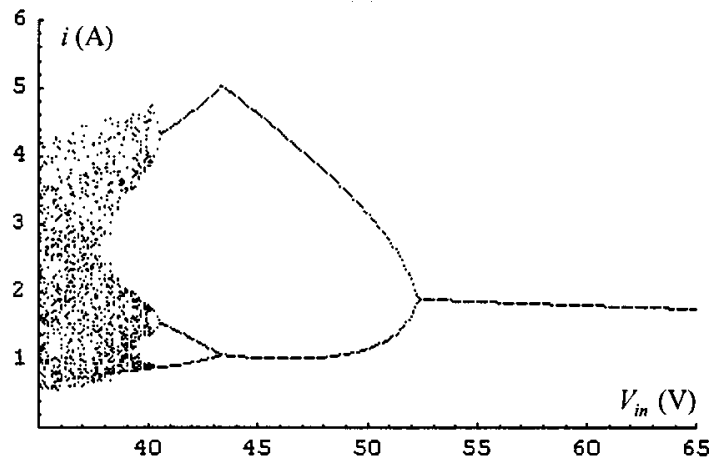

(b)

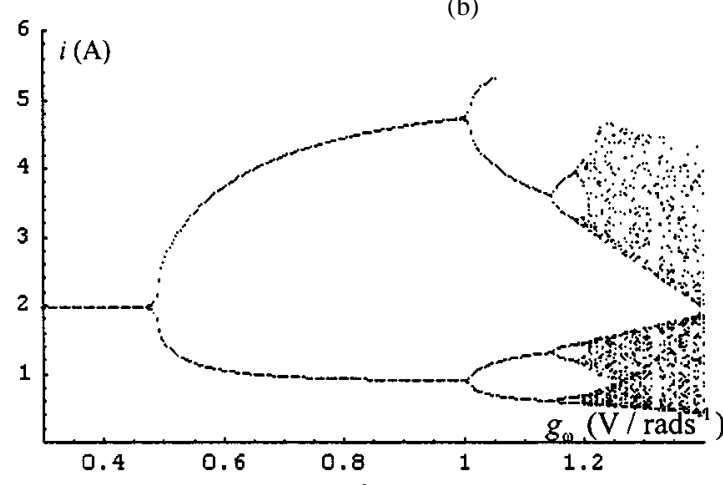

(d)

Fig. 2. Bifurcation diagrams. (a) Speed versus input voltage. (b) Current versus input voltage. (c) Speed versus speed-feedback gain. (d) Current versus speed-feedback gain.

$(0,1)$ for $i=1, \cdots, p, X_{1}^{*}, \cdots, \boldsymbol{X}_{p}^{*}$ can then be obtained from (29) and (30).

Due to the cyclic property of the period-p orbit, $\left\{\boldsymbol{X}_{2}^{*}, \cdots, \boldsymbol{X}_{p}^{*}, \boldsymbol{X}_{1}^{*}\right\}, \cdots,\left\{\boldsymbol{X}_{p}^{*}, \boldsymbol{X}_{1}^{*}, \cdots, \boldsymbol{X}_{p-1}^{*}\right\}$ are other period- $p$ orbits which correspond to the same subharmonic frequency. If $\boldsymbol{X}_{1}^{*}=\cdots=\boldsymbol{X}_{p}^{*}$, the period- $p$ orbit becomes the period-1 orbit, indicating that the period-1 is a particular case of the period- $p$ orbit.

\section{Stability and Characteristic Multipliers}

Both period-1 and period- $p$ orbits may be stable or unstable. Hence, the corresponding characteristic multipliers must be further calculated in order to test the stability of the orbits.

For the period-1 orbit that is a fixed point of the specific Poincaré map given by (25), its characteristic multipliers are eigenvalues of the Jacobian matrix of that mapping, which is given by

$$
\begin{aligned}
D P\left(\boldsymbol{X}^{*}\right) & =\Phi(T)-\frac{\partial \Phi(T-\delta T)}{\partial \delta} \boldsymbol{A}^{-1}\left(\boldsymbol{E}_{1}-\boldsymbol{E}_{2}\right) \frac{\partial \delta}{\partial \boldsymbol{X}^{*}} \\
& =\Phi(T)+\Phi(T-\delta T)\left(\boldsymbol{E}_{1}-\boldsymbol{E}_{2}\right) T \frac{\partial \delta}{\partial \boldsymbol{X}^{*}}
\end{aligned}
$$

where $\left(\partial \delta / \partial \boldsymbol{X}^{*}\right)=((\partial \delta / \partial \omega),(\partial \delta / \partial i))$. According to the implicit-function theorems, $\left(\partial \delta / \partial \boldsymbol{X}^{*}\right)$ can be deduced from (21) as

$$
\begin{aligned}
\frac{\partial \delta}{\partial \boldsymbol{X}^{*}} & =-\left(\frac{\partial h}{\partial \delta}\right)^{-1} \frac{\partial h}{\partial \boldsymbol{X}^{*}} \\
& =-\left[\boldsymbol{E}_{3} \Phi(\delta T)\left(\boldsymbol{A} \boldsymbol{X}^{*}+\boldsymbol{E}_{1}\right) T\right]^{-1} \boldsymbol{E}_{3} \Phi(\delta T)
\end{aligned}
$$

For the period- $p$ orbit, $\left\{X_{1}^{*}, \cdots, X_{p}^{*}\right\}, X_{1}^{*}$ is a fixed point of the $p$-fold iterative specific Poincare map. Therefore, its char-

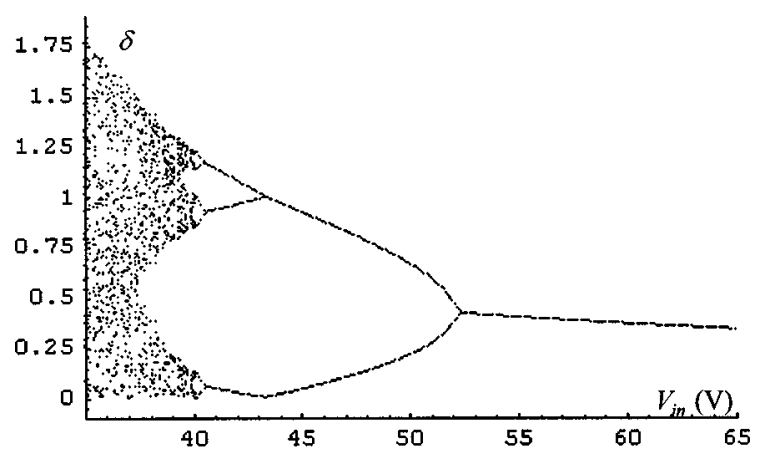

(a)

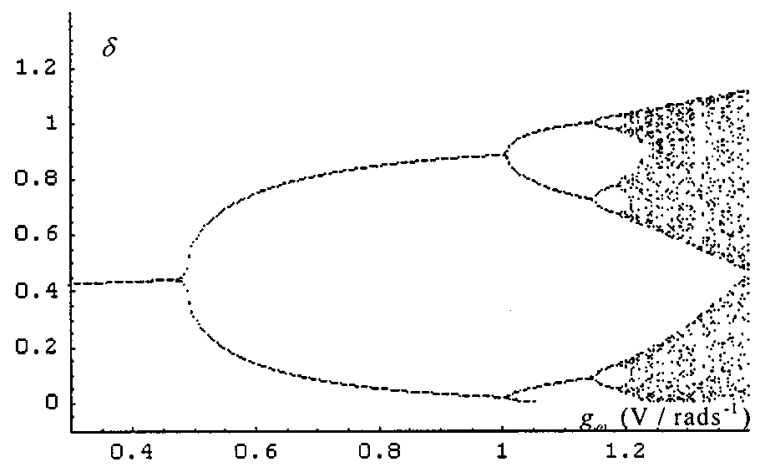

(b)

Fig. 3. Distribution diagrams. (a) Duty cycle versus input voltage. (b) Duty cycle versus speed-feedback gain. 
acteristic multipliers are the eigenvalues of the Jacobian matrix of that mapping, which is given by

$$
\begin{array}{r}
D P^{(p)}\left(\boldsymbol{X}_{1}^{*}\right)=\Phi(p T)+\sum_{j=1}^{p} \Phi((p-j) T) \Phi\left(T-\delta_{j} T\right) \\
\cdot\left(\boldsymbol{E}_{1}-\boldsymbol{E}_{2}\right) T \frac{\partial \delta_{j}}{\partial X_{1}^{*}} .
\end{array}
$$

By substituting (30) into (31), it results that

$$
\boldsymbol{H}\left(\boldsymbol{d}, \boldsymbol{X}_{1}^{*}\right)=\left[h_{1}\left(\boldsymbol{d}, \boldsymbol{X}_{1}^{*}\right), \cdots, h_{p}\left(\boldsymbol{d}, \boldsymbol{X}_{1}^{*}\right)\right]^{\mathrm{T}}=0 .
$$

Hence, according to the implicit-function theorems, the partial derivative in (34) can be expressed as

$$
\frac{\partial \boldsymbol{d}}{\partial \boldsymbol{X}_{1}^{*}}=\left(\frac{\partial \delta_{1}}{\partial \boldsymbol{X}_{1}^{*}}, \cdots, \frac{\partial \delta_{p}}{\partial \boldsymbol{X}_{1}^{*}}\right)^{\mathrm{T}}=-\left[\frac{\partial \boldsymbol{H}}{\partial \boldsymbol{d}}\right]^{-1}\left[\frac{\partial \boldsymbol{H}}{\partial \boldsymbol{X}_{1}^{*}}\right]
$$

where

$$
\begin{aligned}
\frac{\partial \boldsymbol{H}}{\partial \boldsymbol{d}}= & {\left[\frac{\partial h_{i}}{\partial \delta_{j}}\right]_{p \times p} } \\
= & \operatorname{diag}\left[\boldsymbol{E}_{3} \Phi\left(\delta_{i} T\right)\left(\boldsymbol{A} \boldsymbol{X}_{i}^{*}+\boldsymbol{E}_{1}\right) T\right] \\
& +\left[\boldsymbol{E}_{3} \Phi\left(\delta_{\boldsymbol{i}} T\right) \Phi\left((i-j) T-\delta_{j} T\right)\left(\boldsymbol{E}_{1}-\boldsymbol{E}_{2}\right) T\right]_{p \times p}
\end{aligned}
$$

$$
\begin{aligned}
\frac{\partial \boldsymbol{H}}{\partial \boldsymbol{X}_{1}^{*}} & =\left[\frac{\partial h_{1}}{\partial \boldsymbol{X}_{1}^{*}}, \cdots, \frac{\partial h_{p}}{\partial \boldsymbol{X}_{1}^{*}}\right]^{\mathrm{T}} \\
& =\left[\boldsymbol{E}_{3} \Phi\left(\delta_{1} T\right), \cdots, \boldsymbol{E}_{3} \Phi\left((p-1) T+\delta_{p} T\right)\right]^{\mathrm{T}} .
\end{aligned}
$$

Notice that $\Phi\left((i-j) T-\delta_{j} T\right)$ in (37) becomes a zero matrix when $j \geq i$.

\section{COMPUter Simulations}

To illustrate the derived Poincaré map, computer simulations are carried out. The simulation parameters are based on the values of a practical dc drive system, namely, $T=10 \mathrm{~ms}$, $g_{i}=1.1 \mathrm{~V} / \mathrm{A}, g_{\omega}=0.54 \mathrm{~V} / \mathrm{rad} / \mathrm{s}, V_{\text {in }}=50 \mathrm{~V}, R=2.9 \Omega$, $L=53.7 \mathrm{mH}, K_{E}=0.1356 \mathrm{~V} / \mathrm{rad} / \mathrm{s}, K_{T}=0.1324 \mathrm{~N} \cdot \mathrm{m} / \mathrm{A}$, $B=0.000275 \mathrm{~N} \cdot \mathrm{m} / \mathrm{rad} / \mathrm{s}, J=0.000557 \mathrm{~N} \cdot \mathrm{m} / \mathrm{rad} / \mathrm{s}^{2}, T_{l}=$ $0.39 \mathrm{~N} \cdot \mathrm{m}$, and $\omega_{\text {ref }}=105 \mathrm{rad} / \mathrm{s}$. For this set of practical parameters, the resulting eigenvalues of matrix $A$ are $\lambda_{1}=-17.1$ and $\lambda_{2}=-37.4$. It indicates that, although this open-loop system shows no oscillating dynamics, the corresponding closed-loop system may exhibit not only oscillating dynamics, but also subharmonics and even chaos.

\section{A. Bifurcation Diagrams Using Numerical Computation}

By employing the brute-force method to compute the generalized Poincaré map given by (21) and (22), the bifurcation diagrams of $\omega$ and $i$ versus $V_{\text {in }}$ and $g_{\omega}$ can be calculated as shown in Fig. 2. The corresponding $\delta$ with respect to $V_{\text {in }}$ and $g_{\omega}$ are also shown in Fig. 3. As reflected from these figures, the system exhibits chaotic behavior, valid for both chaotic speed and current. It is interesting to note that the period-2 orbit of both $\omega$ and $i$ versus $V_{\text {in }}$ bifurcates to the period-3 orbit when $V_{\text {in }}$ is reduced to $43.4 \mathrm{~V}$, whereas one branch of the period-4 orbit of both $\omega$ and $i$ versus $g_{\omega}$ terminates when $g_{\omega}$ is increased to $1.05 \mathrm{~V} / \mathrm{rad} / \mathrm{s}$. The reason is due to the fact that $\delta$ has to be positive, resulting in the discontinuities at $43.4 \mathrm{~V}$ and $1.05 \mathrm{~V} / \mathrm{rad} / \mathrm{s}$ as shown in Fig. 3.

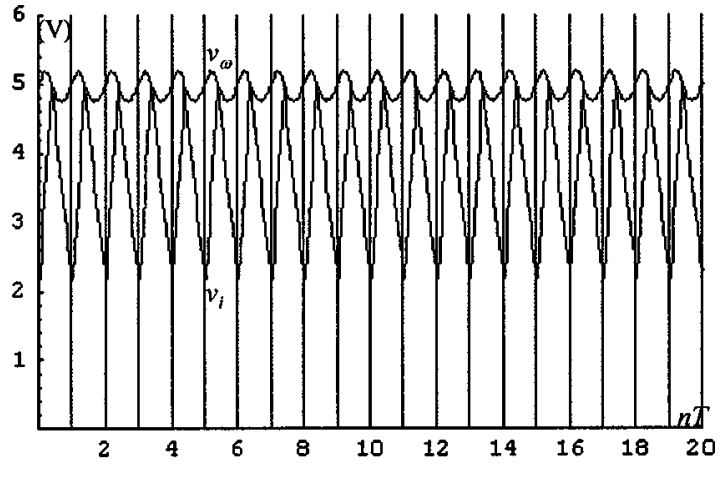

(a)

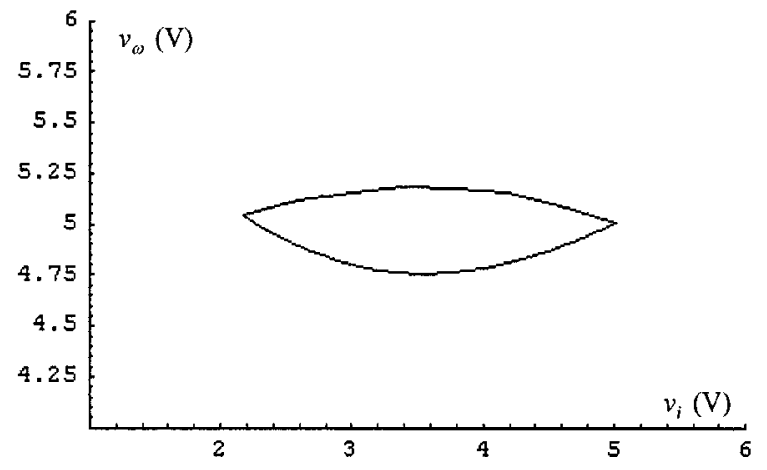

(b)

Fig. 4. Control signals of speed and current during period-1 operation. (a) Waveforms. (b) Trajectory.

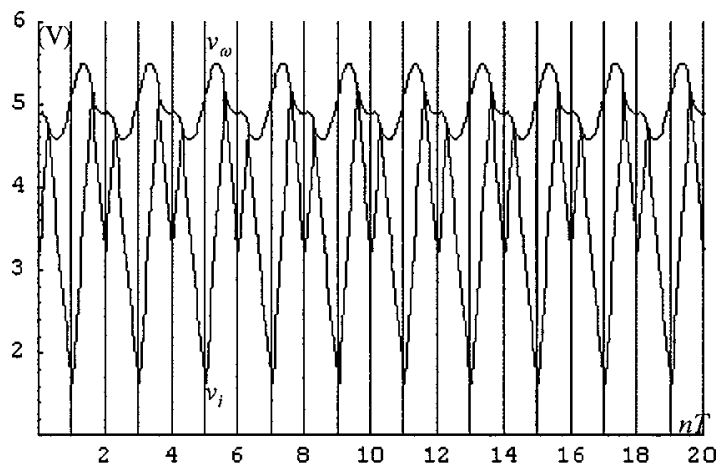

(a)

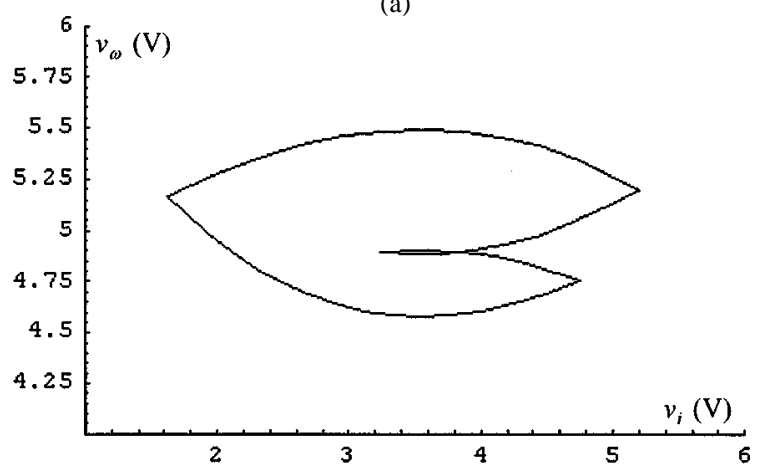

(b)

Fig. 5. Control signals of speed and current during period-2 operation. (a) Waveforms. (b) Trajectory.

The bifurcation diagrams function to illustrate the occurrence of subharmonics and chaos with respect to the variation of 


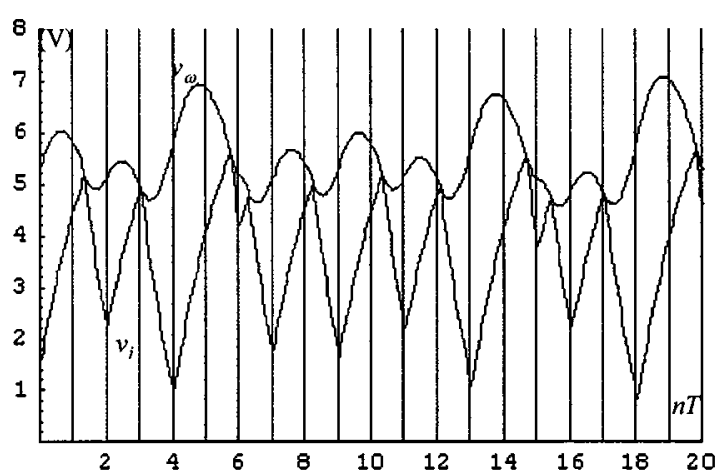

(a)

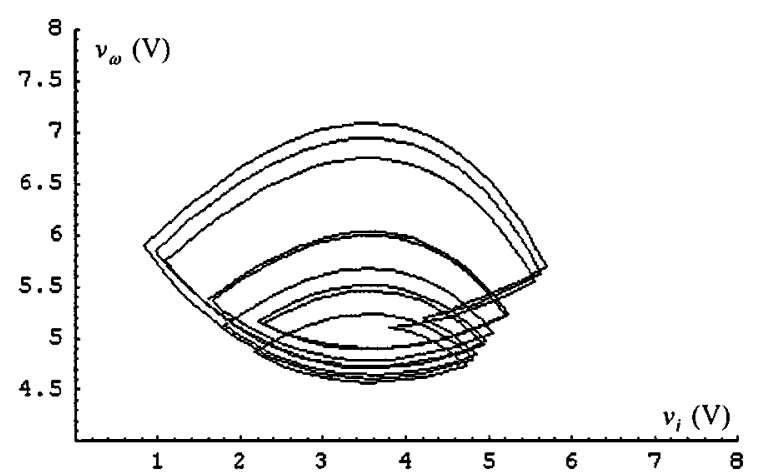

(b)

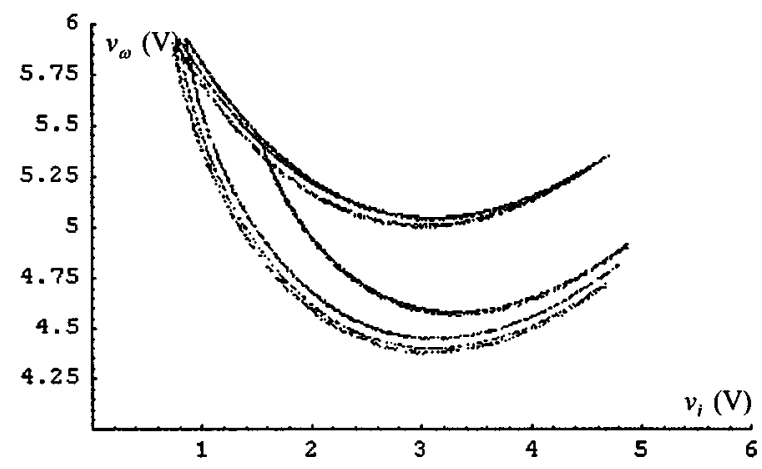

(c)

Fig. 6. Control signals of speed and current during chaotic operation. (a) Waveforms. (b) Trajectory. (c) Poincaré section.

system parameters. In order to attain the insight of subharmonic and chaotic behaviors, both time-domain waveforms and trajectories are investigated. For the sake of clarity, the speed and current control signals, $v_{\omega}$ and $v_{i}$, are used to represent the speed $\omega$ and current $i$, respectively. In fact, they simply obey linear relationships as given by (1) and (2). The simulation waveforms of $v_{\omega}, v_{i}$ and clock pulses as well as the trajectory of $v_{\omega}$ versus $v_{i}$ for the period-1 orbit with $V_{\text {in }}=60 \mathrm{~V}$ and $g_{\omega}=0.54 \mathrm{~V} / \mathrm{rad} / \mathrm{s}$ are shown in Fig. 4. It illustrates that the period-1 trajectory has the boundaries of $v_{i}$ from 2.2 to 5 $\mathrm{V}$ and $v_{\omega}$ from 4.7 to $5.2 \mathrm{~V}$. When $V_{\text {in }}=51 \mathrm{~V}$, the system operates in period-2 orbit, as shown in Fig. 5, in which $v_{i}$ lies between $1.8-5.2 \mathrm{~V}$, while $v_{\omega}$ is between 4.5-5.5 V. Moreover, when $V_{\text {in }}=35 \mathrm{~V}$, the system is in chaos. The corresponding chaotic waveforms and trajectory are shown in Fig. 6(a) and (b), respectively. To further illustrate its chaotic behavior, the system Poincaré section consisting of 4000 sampling points is also shown in Fig. 6(c).

\section{B. Bifurcation Diagrams Using Analytical Modeling}

Based on the derived relationship in (29) to (31) and (34) to (38), the bifurcation diagram of $i$ with respect to $g_{\omega}$, as well as the corresponding duty cycle $\delta$ and characteristic multipliers $\left(\lambda_{1}, \lambda_{2}\right)$ are shown in Fig. 7 in which both the period-1 and period- 2 orbits are involved. It can be found that the system operates in period- 1 when $g_{\omega}<0.48 \mathrm{~V} / \mathrm{rad} / \mathrm{s}$. While $g_{\omega}$ gradually increases to $0.48 \mathrm{~V} / \mathrm{rad} / \mathrm{s}$, one of the magnitudes of $\left(\lambda_{1}, \lambda_{2}\right)$ approaches unity, thus, the system begins to bifurcate to the period-2 orbit. Since one of the duty cycles of the period-2 orbit is equal to unity when $g_{\omega}=1.1 \mathrm{~V} / \mathrm{rad} / \mathrm{s}$, the orbit only exists between 0.48 and $1.1 \mathrm{~V} / \mathrm{rad} / \mathrm{s}$. According to the characteristic multipliers of the period- 2 orbit, it can be found that the period-2 orbit lying $0.48 \mathrm{~V} / \mathrm{rad} / \mathrm{s}<g_{\omega} \leq 1.1 \mathrm{~V} / \mathrm{rad} / \mathrm{s}$ is stable only when $g_{\omega} \leq 1 \mathrm{~V} / \mathrm{rad} / \mathrm{s}$. This bifurcation diagram resulting from analytical modeling closely agrees with that shown in Fig. 2(d) obtained by numerical computation. In fact, without duplicating the figures, other bifurcation diagrams, namely, $i$ versus $V_{\text {in }}$ as well as $\omega$ versus $V_{\text {in }}$ and $g_{\omega}$, can be obtained via analytical modeling and have the same patterns shown in Fig. 2.

It should be noted that the required computational time based on the derived analytical solution is extremely less than that required for computation using the numerical algorithm. Increasingly, the analytical solution can facilitate the identification of the desired stable operating ranges for different system parameters and conditions.

\section{Identification of Stable Operating Ranges}

For a practical dc drive system, the operating point should be designed to locate on the stable period- 1 orbit. The corresponding stability is governed by the period-1 orbit of the specific Poincaré map described by (26) and (27), as well as the characteristic multipliers given by (32) and (33). By substituting $\Phi(\delta T)=1+\delta T A$ into (27), $\delta$ is simply expressed as

$$
\delta=\frac{\boldsymbol{E}_{3}\left(\boldsymbol{A}^{-1} \boldsymbol{E}_{2}+\boldsymbol{E}_{4}\right)}{T \boldsymbol{E}_{3}(\mathbf{1}-\Phi(T))^{-1}\left(\boldsymbol{E}_{1}-\boldsymbol{E}_{2}\right)} .
$$

Due to this explicit expression given by (39), the period-1 orbit and its characteristic multipliers can be easily calculated by using (26), (32), and (33). For a given set of system parameters, the period- 1 orbit is stable if and only if the magnitudes 


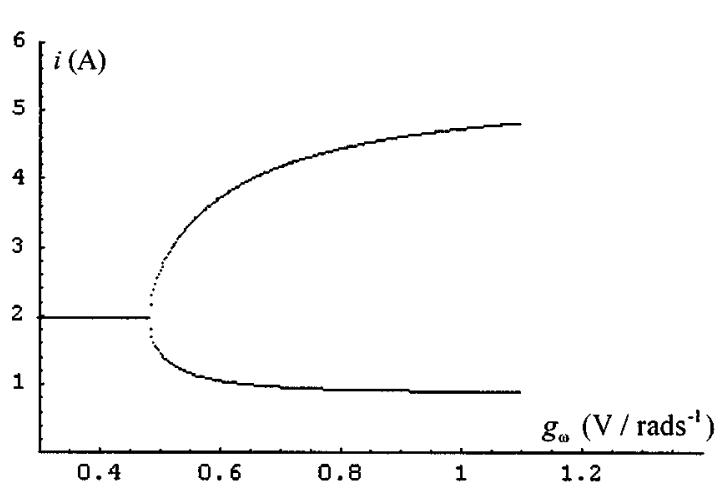

(a)

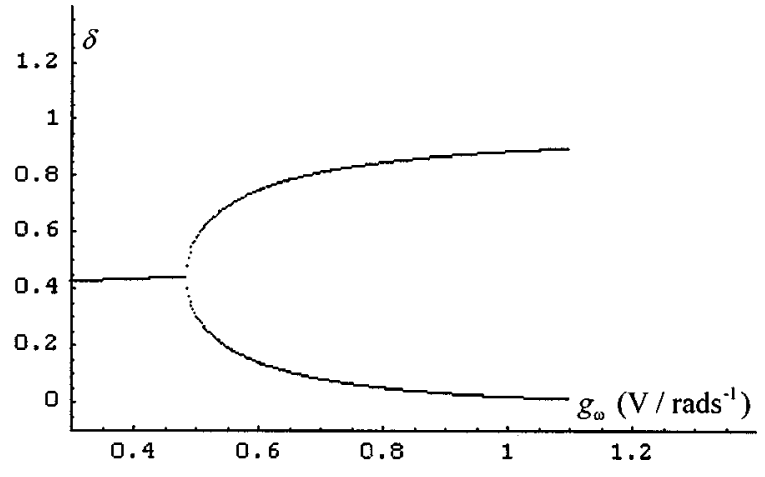

(b)

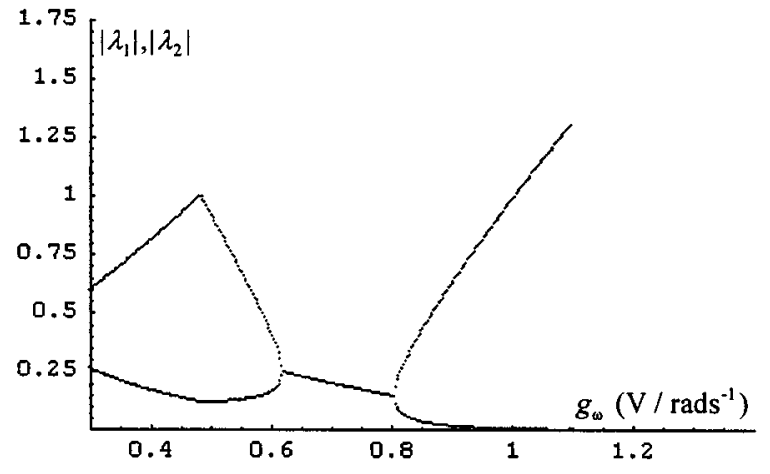

(c)

Fig. 7. Analytical modeling results. (a) Armature current versus speed-feedback gain. (b) Duty cycle versus speed-feedback gain. (c) Characteristic multipliers versus speed-feedback gain.

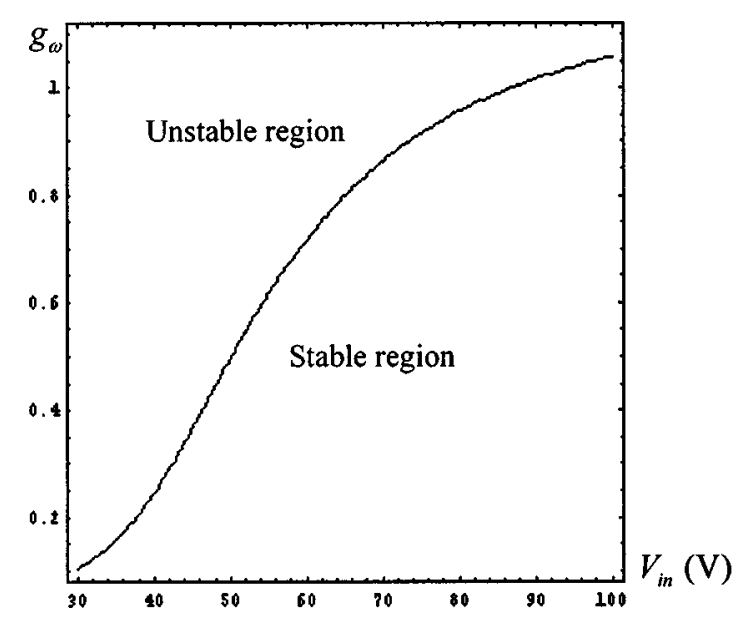

(a)

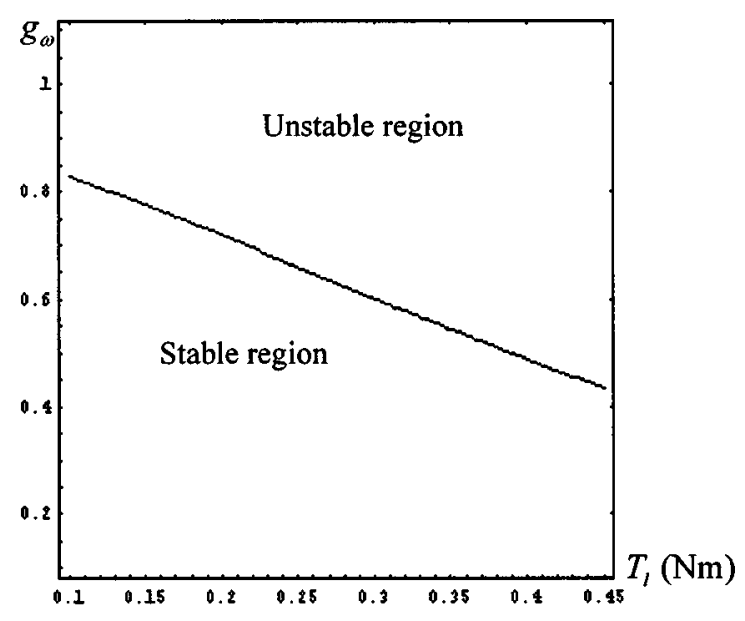

(b)

Fig. 8. Stable regions of typical system parameters. (a) Speed-feedback gain versus input voltage. (b) Speed-feedback gain versus load torque.

of all characteristic multipliers resulting from (32) are less than unity.

Based on the above procedure, the stable operating regions for typical system parameters, namely, $g_{\omega}, V_{\mathrm{in}}$, and $T_{l}$, are determined. As shown in Fig. 8, it indicates that there are different relationships between $g_{\omega}$ and $V_{\text {in }}$, as well as $g_{\omega}$ and $T_{l}$, governing the system stability. Namely, the stable range of the speed feedback gain not only depends on the load torque, but also the input voltage. Stable ranges of other system parameters can similarly be determined by using (26), (32), (33), and (39).

\section{EXPERIMENTAL VERIFICATION}

The experimental setup consists of the current-mode-controlled dc drive system shown in Fig. 1(a). The mechanical load $T_{l}$ is realized by another dc machine which operates in the generator mode. The armature circuit of this machine is then connected to an electronic load which serves as a controllable current sink. Thus, $T_{l}$ can be electronically controlled to keep it at the desired value. As the dc motor and the mechanical load are directly coupled together by a shaft coupling unit, this 

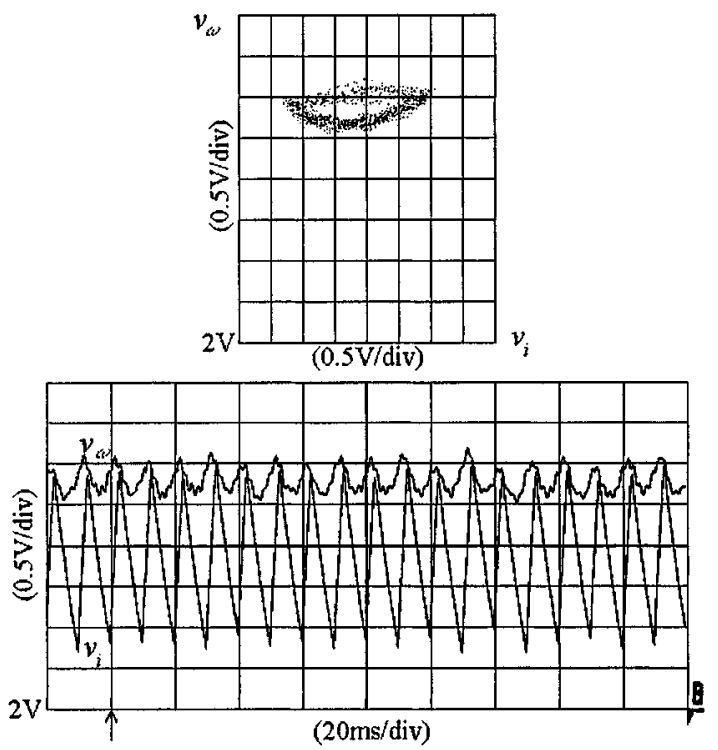

Fig. 9. Measured trajectory and waveforms of speed and current control signals during period-1 operation.
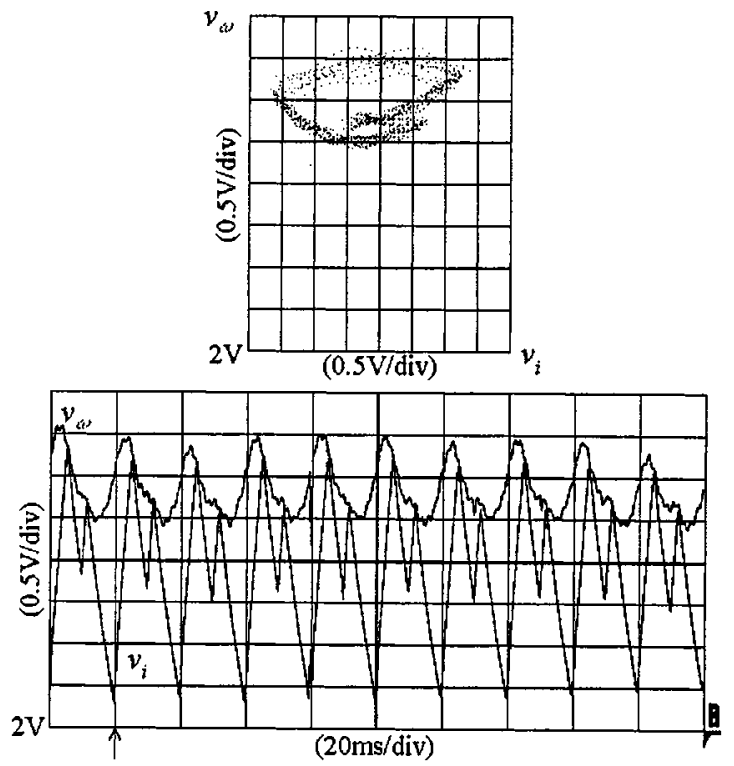

Fig. 10. Measured trajectory and waveforms of speed and current control signals during period-2 operation.

two-mass mechanical system inevitably exhibits mechanical vibration, also called torsional oscillation. Based on the system parameters, the corresponding mechanical resonant frequency is found to be about $1.1 \mathrm{kHz}$ [26]. Since the system switching frequency is selected as $100 \mathrm{~Hz}$, which is much less than the mechanical resonant frequency, the effect of torsional oscillation on the system dynamics is ignored in both theoretical analysis and numerical simulation. As shown in Figs. 9-11, there are high-frequency ripples (about $1.1 \mathrm{kHz}$ ) due to torsional oscillation, superimposing on the measured waveforms of $v_{\omega}$. The magnitude of these ripples is insignificant compared with that of the steady-state periodic solutions.

The measured trajectory and waveforms of $v_{\omega}$ and $v_{i}$ with $V_{\text {in }}=60 \mathrm{~V}$ and $g_{\omega}=0.54 \mathrm{~V} / \mathrm{rad} / \mathrm{s}$ are shown in Fig. 9. It il-

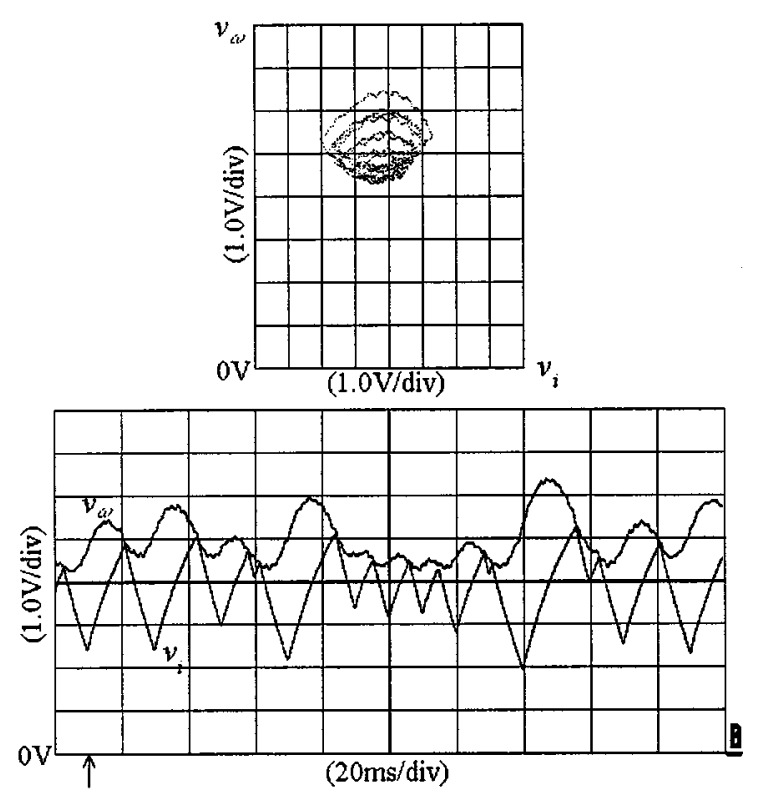

Fig. 11. Measured trajectory and waveforms of speed and current control signals during chaotic operation.

lustrates that the system operates in the period- 1 orbit in which $v_{i}$ lies between 2.7-5 $\mathrm{V}$, while $v_{\omega}$ between 4.7-5.2 $\mathrm{V}$. When $V_{\text {in }}=51 \mathrm{~V}$, the system operates in the period-2 orbit, as shown in Fig. 10, in which $v_{i}$ lies between 2.3-5.2 V, while $v_{\omega}$ between 4.5-5.5 V. Comparing with those shown in Figs. 4 and 5, the measured results and the theoretical prediction have a good agreement.

Moreover, by selecting $V_{\mathrm{in}}=35 \mathrm{~V}$, the measured trajectory and waveforms of $v_{\omega}$ and $v_{i}$ shown in Fig. 11 illustrate that the drive system is in chaotic operation. Different from the period-1 and period- 2 orbits in that the measured trajectory and waveforms are directly compared with the theoretical prediction, the chaotic trajectory and waveforms measured in the experiment can hardly match the theoretical ones because the chaotic behavior is not periodic such that the period of measurement cannot be the same with that of theoretical analysis. Also, its characteristics are extremely sensitive to the system initial conditions. Nevertheless, it can be found that the measured boundaries of the chaotic trajectory shown in Fig. 11 resemble the theoretical prediction in Fig. 6.

\section{CONCLUSIONS}

In this paper, the second-order generalized Poincaré map that describes the nonlinear dynamics of dc drive systems has been derived. Based on the derived map, computer simulations reveal that the system exhibits chaotic behavior. Focusing on the normal operation that the duty cycle is less than unity, the analytical modeling of the period-1 and period- $p$ orbits have also been derived. With the use of these models and the evaluation of characteristic multipliers, the stability analysis can identify the desired stable operating ranges during different system parameters and conditions. The theoretical results have been verified by experimental measurement. Although the exemplification has been focused on a particular dc drive system, the proposed ap- 
proach and derivation can readily be applied or extended to other dc drive systems.

\section{REFERENCES}

[1] R. Tymerski, V. Vorperian, F. C. Y. Lee, and W. T. Baumann, "Nonlinear modeling of the PWM switch," IEEE Trans. Power Electron., vol. 4, pp. 225-233, Apr. 1989.

[2] C. C. Chan and K. T. Chau, "Spectral modeling of switched-mode power converters," IEEE Trans. Ind. Electron., vol. 41, pp. 441-450, Aug. 1994.

[3] D. C. Hamill and D. J. Jefferies, "Subharmonics and chaos in a controlled switched-mode power converter," IEEE Trans. Circuits Syst., vol. 35, pp. 1059-1061, Aug. 1988

[4] J. R. Wood, "Chaos: A real phenomenon in power electronics," in Proc. IEEE APEC'89, 1989, pp. 115-123.

[5] P. T. Krein and R. M. Bass, "Types of instability encountered in simple power electronic circuits: Unboundedness, chattering, and chaos," in Proc. IEEE APEC'90, 1990, pp. 191-194.

[6] J. H. B. Deane and D. C. Hamill, "Instability, subharmonics, and chaos in power electronic systems," IEEE Trans. Power Electron., vol. 5, pp. 260-268, July 1990.

[7] D. C. Hamill, J. H. B. Deane, and D. J. Jefferies, "Modeling of chaotic DC-DC converters by iterated nonlinear mappings," IEEE Trans. Power Electron., vol. 7, pp. 25-36, Jan. 1992.

[8] J. H. B. Deane, "Chaos in a current-mode controlled boost DC-DC converter," IEEE Trans. Circuits Syst. I, vol. 39, pp. 680-683, Aug. 1992.

[9] C. K. Tse, S. C. Fung, and M. W. Kwan, "Experimental confirmation of chaos in a current-programmed Cuk converter," IEEE Trans. Circuits Syst. I, vol. 43, pp. 605-608, July 1996.

[10] E. Fossas and G. Olivar, "Study of chaos in the buck converter," IEEE Trans. Circuits Syst. I, vol. 43, pp. 13-25, Jan. 1996.

[11] K. Chakrabarty, G. Poddar, and S. Banerjee, "Bifurcation behavior of the buck converter," IEEE Trans. Power Electron., vol. 11, pp. 439-447, May 1996.

[12] S. Banerjee, "Coexisting attractors, chaotic saddles, and fractal basins in a power electronic circuit," IEEE Trans. Circuits Syst. I, vol. 44, pp. 847-849, Sept. 1997.

[13] M. di Bernardo, F. Garofalo, L. Glielmo, and F. Vasca, "Switchings, bifurcations, and chaos in DC/DC converters," IEEE Trans. Circuits Syst. $I$, vol. 45, pp. 133-141, Feb. 1998.

[14] G. Poddar, K. Chakrabarty, and S. Banerjee, "Control of chaos in the boost converter," Electron. Lett., vol. 31, no. 11, pp. 841-842, May 1995.

[15] - "Experimental control of chaotic behavior of buck converter," IEEE Trans. Circuits Syst. I, vol. 42, pp. 502-504, Aug. 1995.

[16] J. H. B. Deane and D. C. Hamill, "Improvement of power supply EMC by chaos," Electron. Lett., vol. 32, no. 12, p. 1045, June 1996.

[17] P. J. Aston, J. H. B. Deane, and D. C. Hamill, "Targeting in systems with discontinuities, with applications to power electronics," IEEE Trans. Circuits Syst. I, vol. 44, pp. 1034-1039, Oct. 1997.

[18] Y. Kuroe and S. Hayashi, "Analysis of bifurcation in power electronic induction motor drive systems," in Proc. IEEE PESC'89, 1989, pp. 923-930.

[19] N. Hemati, "Strange attractors in brushless de motors," IEEE Trans. Circuits Syst. I, vol. 41, pp. 40-45, Jan. 1994.

[20] I. Nagy, L. Matakas, Jr., and E. Masada, "Application of the theory of chaos in PWM technique of induction motors," in Proc. Int. Conf. Power Electronics, 1995, pp. 58-63.

[21] K. T. Chau, J. H. Chen, and C. C. Chan, "Modeling of subharmonics and chaos in DC motor drives," in Proc Int. Conf. Industrial Electronics, Control, and Instrumentation, 1997, pp. 523-528.

[22] T. S. Parker and L. O. Chua, "Chaos: A tutorial for engineers," Proc. IEEE, vol. 75 , pp. $982-1008$, Aug. 1987.
[23] - Practical Numerical Algorithms for Chaotic Systems. New York: Springer-Verlag, 1989

[24] S. Wiggins, Introduction to Applied Nonlinear Dynamical Systems and Chaos. New York: Springer-Verlag, 1990.

[25] M. G. Say and E. O. Taylor, Direct Current Machines. London, U.K.: Pitman, 1980

[26] K. Sugiura and Y. Hori, "Vibration suppression in 2- and 3-mass system based on the feedback of imperfect derivative of the estimated torsional torque," IEEE Trans. Ind. Electron., vol. 43, pp. 56-64, Feb. 1996.

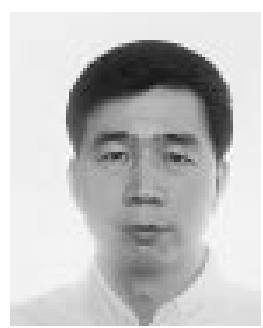

J. H. Chen was born in Guangdong, China. He received the B.Sc. (Eng.) and M.Sc. (Eng.) degrees in electrical engineering from Tsinghua University, Beijing, China, in 1982 and 1987, respectively. He is currently working toward the Ph.D. degree at the University of Hong Kong, Hong Kong.

Prior to entering the Ph.D. program, he was an Associate Professor with the Department of Electrical Engineering, Tsinghua University. His areas of interests include electrical machine and drive design, modeling, simulation, and power electronics. He has authored more than ten published papers in these areas.

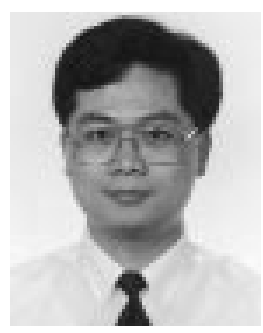

K. T. Chau (M'89) received the first-class honors B.Sc. (Eng.), M.Phil., and Ph.D. degrees in electrical and electronic engineering from the University of Hong Kong, Hong Kong.

He is currently an Associate Professor at the University of Hong Kong. His teaching and research interests focus on three main areas: power converters, machines and drives, and electric vehicles. In these areas, he has published more than 100 refereed technical papers and industrial reports. He has served as chair and organizing committee member for many in-

ternational conferences.

Dr. Chau is listed in Who's Who in Science and Engineering 1998-1999.

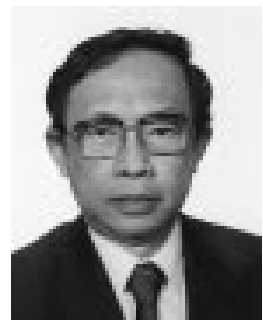

C. C. Chan (M'77-SM'77-F'92) received the B.Sc. degree from China University of Mining and Technology, Beijing, China, the M.Sc. degree from Tsinghua University, Beijing, China, and the Ph.D. degree from the University of Hong Kong, Hong Kong, in 1953,1957 , and 1981 respectively.

He is currently the Head and Honda Chair Professor of Engineering in the Department of Electrical and Electronic Engineering, University of Hong Kong. He has authored four books, published more than 120 technical papers, and holds seven patents. $\mathrm{He}$ is currently the President of the Hong Kong Institution of Engineers.

Prof. Chan is a Fellow of the Royal Academy of Engineering, U.K., a Fellow of the Chinese Academy of Engineering, and a Fellow of the Ukraine Academy of Engineering Science. He was awarded the Honorary D.Sc. degree from the University of Odessa in 1992. He has been very active in the IEEE for more than 20 years. 